\title{
Categorías de áreas protegidas y su uso para el turismo / Un estudio de caso
}

Francisco Orgaz Agüera*

Universidad Tecnológica de Santiago

\section{Resumen}

En los últimos años han aparecido nuevos tipos de turismo alternativos al turismo de masas que se llevan a cabo de forma más sostenible, y muchos de ellos se practican en áreas protegidas. El objetivo de este trabajo es conocer las categorías de áreas protegidas que hay en República Dominicana y sus posibles usos para el turismo. El resultado muestra que ese país cuenta con gran potencial para desarrollar turismo en todas sus categorías de áreas protegidas.

\section{Palabras clave}

República Dominicana, áreas protegidas, recursos naturales, sostenibilidad, turismo.

Recibido: 15/07/2013 - Aceptado: 25/10/2013

*Correo electrónico: franorgaz@ hotmail.com 


\title{
Categories of protected areas and their use for tourism in the Dominican Republic / a Case Study
}

Francisco Orgaz Agüera*

Universidad Tecnológica de Santiago

\begin{abstract}
As opposed to mass tourism, new types of sustainable alternative tourism that are practiced in protected areas have appeared in recent years. The aim of this study is to determine the categories of protected areas present in the Dominican Republic and their possible uses for tourism. The results show that the country has great potential to develop tourism in all it's categories of protected areas.
\end{abstract}

KeY WORDS

Dominican Republic, protected areas, natural resources, sustainability, tourism. 


\section{Introducción}

El turismo se configura como un gran motor económico en el ámbito internacional, que genera nuevas oportunidades para la modernización del destino, tanto en lo económico como en lo social y cultural (Castellanos Verdugo y Orgaz Agüera, 2013).

En la actualidad se realizan de manera sostenible nuevas formas de turismo -alternativas al turismo de masas-, donde el turista tiene en cuenta aspectos como el descanso, el conocimiento de la cultura local y la conservación de los recursos naturales. Esto se debe a que los turistas están más concienciados de las problemáticas medioambientales y a que buscan la autenticidad del destino en su viaje.

Por esta razón, en los últimos tiempos han aparecido diversos tipos de turismo alternativos relacionados con diferentes elementos, como la cultura (Funk y Bruun, 2007; Edelheim, 2009; Tiam Chai, 2011) o la naturaleza (López y Torres, 2009; Jalani, 2012; Zhang y Lai Lei, 2012; Cottrell, Vaske y Roemer, 2013), y que se desarrollan de forma más sostenible que el turismo de masas. Han sido muchos los autores que han estudiado la sostenibilidad en la actividad turística, lo cual ha sido constatado en la investigación desarrollada por Chávez Dagostino, Andrade Romo y Espinosa Sánchez (2013).

Una característica de estas nuevas formas de turismo es que se desarrollan en contacto con la naturaleza y, en ocasiones, en áreas naturales protegidas (ANP), que suelen contar con una legislación para garantizar la adecuada realización de las actividades en ellas, incluido el turismo. Esta normativa es ejecutada por el gobierno del país donde se localiza el área protegida y, según Brenner (2006), se crea con el objetivo de conservar el patrimonio natural mundial.

De acuerdo con Job (2000: 36, cit. en Brenner, 2006: 237), las ANp hacen referencia a los "territorios jurídicamente declaradas para la conservación de la biodiversidad y los recursos naturales. Son áreas de por lo menos 1000 has, pero por lo general más de 10000 has”. Por lo tanto, se excluyen zonas protegidas menores de mil hectáreas (Brenner, 2006).

Así, en las anp se desarrollan diversos tipos de turismo, como el ecoturismo (García-Frapolli et al., 2007; Zhou et al., 2013), el turismo ornitológico (Perdomo y Arias, 2009; Ferrari et al., 2012), el de aventura (Nepal, 2000; Hardiman y Burgin, 2011), el rural (Place, 1991; Nepal, 2008) o el comunitario (Strickland-Munro, 
Allison y Moore, 2010; Bennett et al., 2012), entre otros.

El objetivo de este trabajo es analizar las categorías de áreas protegidas vigentes en República Dominicana y conocer cuáles disponen de uso para el desarrollo del turismo. Se tienen en cuenta todas las áreas protegidas del país, sin considerar, según la definición de Job (2000, cit. en Brenner, 2006), si se trata de un ANP o de un área protegida con menos de mil hectáreas. Una vez analizadas las categorías de áreas protegidas y su posible uso para la actividad turística, se pretende conocer las potencialidades de República Dominicana para desarrollar turismo en sus áreas protegidas.

Para conseguir estos objetivos, el artículo se ha estructurado en seis apartados. Tras esta introducción, en el segundo apartado se hace una presentación sobre el turismo y los recursos naturales en República Dominicana. En el tercero se expone la metodología empleada. En el cuarto se analizan los resultados de la investigación, que muestran las categorías de áreas protegidas vigentes en República Dominicana, sus posibles usos para el turismo y sus potencialidades turísticas en general. En el quinto se ofrecen las conclusiones del estudio. En el último se incluye la bibliografía utilizada en la investigación.

\section{República Dominicana, turismo y recursos naturales}

República Dominicana es uno de los principales destinos internacionales de turismo de sol y playa y ha sido objeto de estudio de diversos investigadores (Moreno Gil, Celis Sosa y Aguilar Quintana, 2002; Villarreal y Van der Horst, 2008; Agosín et al., 2009; Orgaz Agüera, 2012; Castellanos Verdugo y Orgaz Agüera, 2013). El turismo es su industria más importante (Villarreal y Van der Horst, 2008). De acuerdo con Moreno Gil, Celis Sosa y Aguilar Quintana (2002), destaca el turismo de sol y playa realizado en hoteles resort en régimen de "todo incluido”, y según Castellanos Verdugo y Orgaz Agüera (2013), son escasas las ofertas complementarias.

Agosín et al. (2009) señalan que en República Dominicana existen seis polos turísticos. El primero se encuentra en la región este del país, donde se localizan Punta Cana-Bávaro y La Romana-Bayahibe; aquí se ubica el principal destino de turismo de sol y playa, además del recurso natural más visitado de la nación: Isla Saona, en el Parque Nacional del Este. El segundo polo turístico se localiza 
en Santo Domingo, destacado por viajeros de negocios, aunque también se desarrolla, en menor medida, turismo de sol y playa en la zona de Boca Chica y Juan Dolio. El tercer polo turístico se halla en el norte del país, en concreto en la provincia de Puerto Plata, donde predomina el turismo de sol y playa (segundo destino en esta tipología) y el turismo deportivo (Cabarete), pero también se encuentra otro de los recursos naturales más visitados: el monumento natural Saltos de la Damajagua, donde se puede practicar el ecoturismo y el turismo activo. En el centro del país se ubica el cuarto polo turístico, el principal destino de montaña, en las ciudades de Constanza y Jarabacoa; aquí se localiza la zona más alta de todo el Caribe: el Pico Duarte. El quinto polo turístico está en la zona noreste, en la península de Samaná, donde sobresalen tres tipos de turismo: de sol y playa, de cruceros y de segunda residencia. Por último, en el sur de la nación se sitúa el polo con más potencial ecoturístico, pero, según Agosín et al. (2009), aún no está lo suficientemente desarrollado.

Estos polos turísticos fueron visitados por más de cuatro millones de turistas en 2012, lo que confirma el constante aumento de turistas que está recibiendo República Dominicana. Estos destinos cuentan con más de 65000 habitaciones, pertenecientes la mayoría de ellas a cadenas hoteleras extranjeras dominantes en el país (Agosín et al., 2009).

En cuanto a los recursos naturales, Castellanos Verdugo y Orgaz Agüera (2013) afirman que República Dominicana posee gran potencial para poner en marcha actividades ecoturísticas complementarias al turismo de sol y playa, aprovechando para ello sus numerosas áreas protegidas.

Teniendo en cuenta que el principal turismo del país es el de sol y playa, los recursos naturales nacionales más importantes son el agua cristalina, la vegetación exuberante y la arena blanca y fina de las playas, las cuales están bañadas por el mar Caribe al sur y el este del país, y por el océano Atlántico al norte.

\section{Metodología}

La metodología empleada en este trabajo fue una combinación de fuentes primarias y secundarias. Las primeras consistieron en técnicas cualitativas en el destino objeto de estudio: entrevistas, grupos de discusión y observación parti-

126 cipante, con la finalidad de conocer los aspectos más relevantes de los recursos 
naturales y las áreas protegidas, entre ellos sus normativas referentes al turismo. Las segundas constituyeron una revisión de la literatura acerca del turismo en áreas protegidas, así como de leyes y otras normativas sobre turismo, medio ambiente y recursos naturales establecidas en República Dominicana. También se consultaron fuentes secundarias estadísticas para conocer los principales datos sobre turismo del país.

Las entrevistas son un acto de comunicación oral entre dos o más personas, con el fin de obtener información (Malhotra, 2004). En este estudio se efectuaron con personal involucrado en la gestión y planificación del turismo y las áreas protegidas dominicanas, y se llevaron a cabo de enero a mayo de 2013.

A su vez, el grupo de discusión es una técnica de investigación en la que se reúnen entre cinco y ocho personas para expresar su visión sobre un tema dado mediante una conversación en un ambiente permisivo, nunca directivo (Krueger, 1991). Esta técnica se realizó con profesionales que trabajan en las áreas protegidas del país o que tienen conocimiento sobre el desarrollo del turismo nacional.

Por último, la observación participante consiste en "observar" in situ los principales aspectos que se están estudiando, a través de la inclusión en "vivencias” con los agentes locales (Orgaz Agüera, 2012); en este caso se visitaron los principales polos turísticos dominicanos y las áreas protegidas con potencialidades turísticas.

La entrevista, los grupos de discusión y la observación participante se realizaron de mayo a agosto de 2011, de junio a septiembre de 2012 y de enero a mayo de 2013. En todo momento, estas técnicas se hicieron previa autorización de los participantes o agentes implicados, transcribiendo posteriormente los datos obtenidos a un documento.

Las entrevistas fueron efectuadas por dos personas, lo cual resultó fundamental por varias razones (Caro González, Castellanos Verdugo y Velázquez González, 2006): porque el otro entrevistador era originario del país objeto de la investigación y experto en áreas protegidas; porque facilitó la interacción con el entrevistado; porque permitió prestar más atención a todo el proceso de comunicación verbal y no verbal que se establecía, y porque ayudó a reducir la subjetividad de la percepción de un único entrevistador.

Una vez obtenida la información tanto de las fuentes primarias como de las secundarias, se analizan y exponen los principales resultados de la investigación. 


\section{Resultados}

República Dominicana cuenta con un Sistema Nacional de Áreas Protegidas (Sinap) que está regulado por la Ley Sectorial No. 202-04 de Áreas Protegidas. ${ }^{1}$ En este documento se citan seis categorías de manejo de áreas protegidas que a su vez se dividen en 13 subcategorías. Esta ley es posterior a la ley de medio ambiente (Ley General No. 64-00 sobre Medio Ambiente y Recursos Naturales de República Dominicana, del año 2000)² que regula todo lo relacionado con las áreas protegidas, siguiendo las bases marcadas por la ley sobre medio ambiente y recursos naturales.

En el cuadro 1 se observa la denominación de las diferentes categorías y subcategorías que existen en República Dominicana según la Ley Sectorial No. 202-04 de Áreas Protegidas.

\section{Cuadro 1. Categorías de áreas protegidas en República Dominicana}

\section{CATEgoría}

I. Áreas de protección estrictas

II. Parques nacionales

III. Monumentos naturales

IV. Áreas de manejo de hábitat/especies

V. Reservas naturales

VI. Paisajes protegidos

\section{SubCATEgoría}

Reserva científica

Santuario de mamíferos marinos

Parque nacional

Parque nacional submarino

Monumento natural

Monumento cultural

Refugio de vida silvestre

Reservas forestales

Bosque modelo

Reserva privada

Vías panorámicas

Corredor biológico

Áreas nacionales de recreo

Fuente: Elaboración propia.

${ }^{1}$ Ley 202 de 2004. Ley Sectorial de Áreas Protegidas < http://www.jmarcano.com/ecohis/ legales/ley202.html > [2013, 2 de junio].

${ }^{2}$ Ley 64 de 2000. Ley General sobre Medio Ambiente y Recursos Naturales < http://www. jmarcano.com/ecohis/legales/ley64.html > [2013, 21 de junio $]$. 
Según la Ley Sectorial No. 202-04 de Áreas Protegidas, República Dominicana cuenta dentro del Sinap con seis categorías de áreas protegidas y 13 subcategorías. Por su parte, en el Atlas de biodiversidad y recursos naturales de la República Dominicana, elaborado por el Ministerio de Medio Ambiente y Recursos Naturales de República Dominicana (Reyna Alcántara y Polonia Martínez, 2012), se mencionan las mismas seis categorías, pero se modifican algunas subcategorías.

En el cuadro 2 se muestran las categorías y subcategorías de manejo de áreas protegidas recogidas por el Atlas de biodiversidad y recursos naturales de la República Dominicana.

Cuadro 2. Categorías y subcategorías de áreas protegidas

\begin{tabular}{ll}
\multicolumn{1}{c}{ CATEGoría } & \multicolumn{1}{c}{ SubCATEGoRía } \\
\hline I. Áreas de protección estricta & $\begin{array}{l}\text { Reservas científicas } \\
\text { Santuarios de mamíferos marinos } \\
\text { Reserva biológica }\end{array}$ \\
II. Parques nacionales & $\begin{array}{l}\text { Parques nacionales } \\
\text { Parques nacionales submarinos }\end{array}$ \\
III. Monumentos naturales & $\begin{array}{l}\text { Monumentos naturales } \\
\text { Refugios de vida silvestre }\end{array}$ \\
IV. Áreas de manejo de hábitat/especies & $\begin{array}{l}\text { Refugios de vida silvestre } \\
\text { V. Reservas naturales }\end{array}$ \\
VI. Paisajes protegidos & Reservas forestales \\
& Vía panorámica \\
& $\begin{array}{l}\text { Áreas naturales de recreo } \\
\text { Corredor ecológico }\end{array}$
\end{tabular}

Fuente: Elaboración propia a partir de Reyna Alcántara y Polonia Martínez (2012).

En la categoría I (Áreas de protección estricta), el Ministerio de Medio Ambiente y Recursos Naturales añade la subcategoría Reservas biológicas, inexistente en la Ley Sectorial No. 202-04 de Áreas Protegidas. En la categoría II (Parques nacionales) no se observa ningún cambio entre lo que aparece en la Ley Sectorial No. 202-04 de Áreas Protegidas y el Atlas de biodiversidad y recursos 
naturales. La categoría III (Monumentos naturales) tiene en la ley las subcategorías Monumento natural y Monumento cultural, y, en el Atlas, la categoría de Monumento cultural es modificada por la de Refugios de vida silvestre. La categoría IV (Áreas de manejo de hábitat/especies) no cambia, y mantiene una sola subcategoría, si bien esta categoría, denominada Refugio de vida silvestre, coincide con una de las establecidas en la categoría de manejo anterior. La categoría V (Reservas naturales) pasa de tres subcategorías en la ley (Reservas forestales, Bosque modelo y Reserva privada) a una en el Atlas de biodiversidad y recursos naturales (Reservas forestales). Por último, la categoría VI (Paisajes protegidos) se mantiene de la misma forma, aunque la subcategoría denominada Áreas nacionales de recreo cambia a Áreas naturales de recreo.

Las seis categorías de áreas protegidas que posee República Dominicana están definidas en el artículo 2 de la Ley Sectorial No. 202-04 de Áreas Protegidas de la siguiente manera:

I. Área de protección estricta: Área terrestre y/o marina de extensión variable que posee ecosistemas representativos o excepcionales con características geológicas o fisiográficas y biológicas particulares y/o especies de interés singular para investigaciones científicas y/o monitoreo ambiental con ninguna o muy poca presencia o actividades humanas y que, por su singularidad o rareza, requieren de un manejo restringido.

II. Parque nacional: Área natural terrestre y/o marina designada para:

1. Proteger la integridad ecológica de uno o más ecosistemas con cobertura boscosa o sin ella para provecho de las presentes y futuras generaciones.

2. Excluir explotaciones y ocupaciones intensivas que alteren sus ecosistemas.

3. Proveer la base para crear las oportunidades de esparcimiento espiritual, de actividades científicas, educativas, recreacionales y turísticas, considerando inversiones necesarias para ello.

III. Monumento natural: Áreas que contengan uno o más rasgos naturales específicos o naturales-culturales que posean un valor sobresaliente o único debido a su rareza intrínseca, a sus cualidades estéticas representativas o a su significación natural-cultural. Esto incluye cavernas y cuevas, o áreas con monumentos 
o ruinas de interés histórico.

IV. Áreas de manejo de hábitat/especies: Una porción de terreno dedicada a la protección y mantenimiento de biodiversidad y recursos naturales donde existen diferentes especies -conjunto de organismos capaces de reproducirse entre sí- y hábitat -lugar o ambiente donde existen naturalmente un organismo o una población.

V. Reserva natural: Áreas no modificadas o ligeramente modificadas, o tierras o aguas que mantienen sus características e influencias naturales sin poblaciones humanas permanentes o significativas y que son para conservar sus condiciones naturales.

VI. Paisaje protegido: Área terrestre, marina y/o costero-marina donde la interacción de las poblaciones humanas y la naturaleza han producido un área de carácter diferente, con un valor estético, cultural y/o ecológico significativo y de alta diversidad biológica.

Existen 123 áreas protegidas en República Dominicana clasificadas en estas seis categorías de manejo del Sinap. Según Reyna Alcántara y Polonia Martínez (2012), las 123 unidades de conservación se distribuyen así:

- Áreas de protección estricta: 12

- Parques nacionales: 31

- Monumentos naturales: 30

- Áreas de manejo de hábitat/especies: 19

- Reservas naturales: 15

- Paisajes protegidos: 16

Como puede apreciarse, destacan sobre todo los parques nacionales y los monumentos naturales, seguidos por las áreas de manejo de hábitat/especies. En menor medida se encuentran los paisajes protegidos, las reservas naturales y las áreas de protección estricta.

De acuerdo con el artículo 14 de la Ley Sectorial No. 202-04 de Áreas Protegidas, cada categoría tiene diferentes objetivos de manejo y uso permitido. En el cuadro 3 se observan las seis categorías de manejo de áreas protegidas dominicanas, además de sus objetivos de manejo y usos permitidos en el terreno localizado dentro de los límites del área protegida. 
Análisis de las categorías de áreas protegidas y su uso para el turismo / Un estudio de caso

Cuadro 3. Objetivos de manejo y uso permitido en las categorías de áreas protegidas

\begin{tabular}{|c|c|c|}
\hline Categoría & OBJetivos DE MANEJo & USOS PERMITIDOS \\
\hline $\begin{array}{l}\text { I. Áreas de } \\
\text { protección estricta }\end{array}$ & $\begin{array}{l}\text { Proteger los recursos naturales } \\
\text { y naturales singulares }\end{array}$ & $\begin{array}{l}\text { Investigación científica, monitoreo, } \\
\text { educación, conservación de los } \\
\text { recursos genéticos, turismo ecológico } \\
\text { y desarrollo de infraestructura desti- } \\
\text { nada a la protección }\end{array}$ \\
\hline II. Parques nacionales & $\begin{array}{l}\text { Resguardar todos los ecosiste- } \\
\text { mas del área protegida, evitan- } \\
\text { do explotaciones y ocupacio- } \\
\text { nes que alteren ecosistemas, } \\
\text { y crear oportunidades para el } \\
\text { desarrollo del ocio sostenible }\end{array}$ & $\begin{array}{l}\text { Investigación científica, educación, } \\
\text { recreación, ecoturismo y desarrollo } \\
\text { de infraestructura para la protección } \\
\text { del medio natural y para la realiza- } \\
\text { ción de actividades ecoturísticas }\end{array}$ \\
\hline $\begin{array}{l}\text { III y IV. Área de } \\
\text { protección especial }\end{array}$ & $\begin{array}{l}\text { Proteger elementos naturales } \\
\text { con importantes componentes } \\
\text { bióticos, estéticos y culturales }\end{array}$ & $\begin{array}{l}\text { Investigación científica, educación, } \\
\text { recreación, ecoturismo y desarro- } \\
\text { llo de infraestructura para ocio y } \\
\text { recreación, para uso público y para } \\
\text { ecoturismo }\end{array}$ \\
\hline V. Reserva natural & $\begin{array}{l}\text { Garantizar la conservación de } \\
\text { flora y fauna }\end{array}$ & $\begin{array}{l}\text { Aprovechamiento controlado de } \\
\text { recursos naturales, educación, } \\
\text { recreación, ecoturismo, desarrollo de } \\
\text { actividades tradicionales y creación } \\
\text { de infraestructura sostenible }\end{array}$ \\
\hline VI. Paisajes protegidos & $\begin{array}{l}\text { Mantener paisajes con caracte- } \\
\text { rísticas de la interacción entre } \\
\text { el hombre y la naturaleza, con- } \\
\text { servar el patrimonio cultural y } \\
\text { natural y generar beneficios a } \\
\text { través de actividades sosteni- } \\
\text { bles y ecoturismo }\end{array}$ & $\begin{array}{l}\text { Recreación, turismo, actividades eco- } \\
\text { nómicas, infraestructura de vivienda } \\
\text { y para usos turísticos y tradicionales } \\
\text { del suelo }\end{array}$ \\
\hline
\end{tabular}

Fuente: Elaboración propia. 
Siguiendo el cuadro 3, se debe tener en cuenta que la Ley Sectorial No. 20204 de Áreas Protegidas incluye a la categoría III (Monumentos naturales) y a la IV (Áreas de manejo de hábitat/especies) en el grupo de Área de protección especial.

En cuanto a los diferentes usos permitidos en las áreas protegidas dominicanas, se pueden desarrollar, bajo los principios sostenibles marcados por el Ministerio de Medio Ambiente y Recursos Naturales, actividades turísticas o ecoturísticas en todas las categorías de protección. En concreto, los usos relacionados con el turismo en cada categoría de área protegida son:

- Áreas de protección estricta: turismo ecológico

- Parques nacionales: actividades ecoturísticas

- Monumentos naturales: ecoturismo

- Áreas de manejo de hábitat/especies: ecoturismo

- Reserva natural: ecoturismo

- Paisajes protegidos: usos turísticos

Los usos vinculados con el turismo en las áreas protegidas de República Dominicana tienen que ver con la tipología del ecoturismo, caracterizada como una forma de turismo sostenible que busca mejorar el desarrollo socioeconómico de las comunidades locales y conservar los recursos naturales para su uso por las generaciones futuras. No obstante, en otros estudios se constata que en un gran porcentaje de áreas protegidas no se realizan estas actividades ecoturísticas (Orgaz Agüera, 2012; Castellanos Verdugo y Orgaz Agüera, 2013). Por tanto, de acuerdo con Castellanos Verdugo y Orgaz Agüera (2013), existe gran potencial para poner en marcha actividades ecoturísticas en las áreas protegidas dominicanas.

En este sentido, las potencialidades destacadas por el trabajo de campo de esta investigación, localizadas en las áreas protegidas o en los límites de las mismas, son: avistamiento de flora y fauna; senderismo; baño en ríos y balnearios naturales; paseo en bicicleta, en botes o kayaks; actividades de degustación de productos gastronómicos, y visitas a zonas patrimoniales y culturales.

Estas potencialidades se pueden clasificar en varias formas de turismo: ecoturismo, turismo activo, ornitológico (birdwatching) y gastronómico. En este aspecto, se observa un modo de explotar estos tipos de turismo de forma adecuada a 
través de lo que se denomina turismo comunitario, es decir, que las comunidades locales tengan un gran peso en la gestión y planificación de las actividades 0 productos turísticos en las áreas protegidas y, por ende, sean beneficiarias de esta actividad.

\section{Conclusiones}

El turismo se está configurando como uno de los principales motores económicos internacionales y, con la puesta en marcha de las nuevas formas de turismo sostenibles, está generando efectos positivos en el destino, que se traducen en el desarrollo socioeconómico de las comunidades locales y en la conservación y el respeto hacia los recursos naturales y la cultura local.

Muchas de esas nuevas formas de turismo se llevan a cabo en zonas naturales, de las cuales buena parte son áreas protegidas. En República Dominicana se establecen, a través del Sinap, un total de seis categorías, que coinciden con las mencionadas por la Unión Internacional para la Conservación de la Naturaleza (uicn).

La ley que regula el uso de estas áreas es la Ley Sectorial No. 202-04 de Áreas Protegidas, aprobada en 2004, bajo las premisas de la Ley General No. 64-00, del año 2000, sobre Medio Ambiente y Recursos Naturales. En la ley sobre áreas protegidas se dispone que en todas las categorías de áreas protegidas del país se pueden desarrollar actividades turísticas o ecoturísticas, siempre y cuando se realicen de forma sostenible.

República Dominicana cuenta actualmente con 123 áreas protegidas, clasificadas en seis áreas de manejo, pero no todas se aprovechan para realizar actividades turísticas o ecoturistas, ya sea dirigidas a los residentes dominicanos o a extranjeros; sin embargo, muchas de estas áreas protegidas tienen gran potencial para el turismo y se localizan en una posición estratégica, es decir, cerca de los grandes polos turísticos del país y de los aeropuertos en que transitan más turistas durante el año.

Entonces, aunque la ley permite el turismo o ecoturismo en las áreas protegidas dominicanas, y estas cuentan con diversas potencialidades para ello (ecoturismo, turismo gastronómico, ornitológico y activo), la administración pública con competencia para diseñar productos o actividades turísticas en es- 
tas áreas no aprovecha tal potencial; además, estas actividades ayudarían a mejorar el desarrollo socioeconómico de las comunidades locales, pues fomentarían el nacimiento de varias empresas y la creación de empleos, y contribuirían a minimizar los índices de pobreza en las zonas cercanas a una gran cantidad de áreas protegidas.

\section{Fuentes consultadas}

Agosín, M. et al. (eds.) (2009). La ruta hacia el crecimiento sostenible en la República Dominicana: Fiscalidad, competitividad, institucionalidad y electricidad. Santo Domingo: Banco Interamericano de Desarrollo.

Bennett, N. et al. (2012). "A Capital Assets Framework for Appraising and Building Capacity for Tourism Development in Aboriginal Protected Area Gateway Communities”. Tourism Management, 33 (4), 752-766.

Brenner, L. (2006). “Áreas naturales protegidas y ecoturismo: el caso de la Reserva de la Biosfera Mariposa Monarca, México”. Relaciones: Estudios de Historia y Sociedad, 27 (105), 237-265.

Caro González, F.J., M. Castellanos Verdugo y J.L. Velázquez González (2006). "Agentes sociales, turismo y espacios urbanos: Una aplicación de la teoría fundamentada en investigaciones de turismo”, en Actas del $X$ Congreso AECIT: Turismo y territorio: conflictos, corresponsabilidad y estrategias de gestión. Bilbao: Asociación Española de Expertos Científicos en Turismo, 1-11.

Castellanos Verdugo, M. y F. Orgaz Agüera (2013). "Potencialidades ecoturísticas de la República Dominicana”. TURyDES: Revista de Investigación en Turismo y Desarrollo Local, 6 (14), 1-15.

Chávez Dagostino, R.M., E. Andrade Romo y R. Espinosa Sánchez (2013). “Turismo y desarrollo sustentable: contribución de Hispanoamérica”. Teoría y Praxis, 13, 9-33.

Cottrell, S.P., J.J. Vaske y J.M. Roemer (2013). "Resident Satisfaction with Sustainable Tourism: The Case of Frankenwald Nature Park, Germany". Tourism Management Perspectives, 8, 42-48.

Edelheim, J.R. (2009). "With the Simpsons as Tour Guides: How Popular Culture Sources can Enhance the Student Experience in a University Tourism 
Unit”. Journal of Hospitality and Tourism Management, 16 (1), 113-119. Ferrari, S. et al. (2012). "Turismo y aves playeras migratorias en la Patagonia Austral (Santa Cruz, Argentina): Lineamientos para minimizar el disturbio humano y ordenar la actividad". TURyDES: Revista de Investigación en Turismo y Desarrollo Local, 5 (13), 1-15.

Funk, D.C. y T.J. Bruun (2007). “The Role of Socio-Psychological and CultureEducation Motives in Marketing International Sport Tourism: A CrossCultural perspective”. Tourism Management, 28 (3), 806-819.

García-Frapolli, E. et al. (2007). "Biodiversity Conservation, Traditional Agriculture and Ecotourism: Land Cover/Land Use Change Projections for a Natural Protected Area in the Northeastern Yucatan Peninsula, Mexico". Landscape and Urban Planning, 83 (2-3), 137-153.

Hardiman, N. y S. Burgin (2011). "Canyoning Adventure Recreation in the Blue Mountains World Heritage Area (Australia): The Canyoners and Canyoning Trends over the Last Decade”. Tourism Management, 32 (6), 1324-1331.

Jalani, J.O. (2012). "Local People's Perception on the Impacts and Importance of Ecotourism in Sabang, Palawan, Philippines”. Procedia: Social and Behavioral Sciences, 57, 247-254.

Krueger, R. (1991). El grupo de discusión: Guía práctica de la investigación aplicada. Madrid: Pirámide.

López, M. y C. Torres (2009). "Redes sociales en proyectos ecoturísticos”. Teoría $y$ Praxis, 7, 101-114.

Malhotra, N.K. (2004). "Introducción y primeras fases de la investigación de mercados”, en Investigación de mercados. Un enfoque aplicado. México: Prentice Hall, 1-70.

Moreno Gil, S., D.F. Celis Sosa y T. Aguilar Quintana (2002). “Análisis de la satisfacción del turista de paquetes turísticos respecto a las actividades de ocio en el destino: El caso de República Dominicana”. Cuadernos de Turismo, 9, 67-84.

Nepal, S.K. (2000). "Tourism in Protected Areas: The Nepalese Himalaya”. Annals of Tourism Research, 27 (3), 661-681.

- (2008). “Tourism-Induced Rural Energy Consumption in the Annapurna Region of Nepal”. Tourism Management, 29 (1), 89-100. 
Orgaz Agüera, F. (2012). "Potencialidades del turismo ecológico en República Dominicana: Diseño y creación de la eco-ruta Macorix”, en Turismo y sostenibilidad: V Jornadas de Investigación en Turismo en Universidad de Sevilla. Sevilla: Ministerio de Medio Ambiente y Recursos Naturales, 487-513.

Perdomo, L. y Y. Arias (2009). Important Bird Areas Americas: Dominican Republic. Quito: Bird Life International.

Place, S.E. (1991). "Nature Tourism and Rural Development in Tortuguero". Annals of Tourism Research, 18 (2), 186-201.

Reyna Alcántara, E. y A. Polonia Martínez (2012). Atlas de biodiversidad y recursos naturales de la República Dominicana. Santo Domingo: Ministerio de Medio Ambiente y Recursos Naturales.

Strickland-Munro, J.K., H.E. Allison y S.A. Moore (2010). “Using Resilience Concepts to Investigate the Impacts of Protected Area Tourism on Communities". Annals of Tourism Research, 37 (2), 499-519.

Tiam Chai, L. (2011). "Culture Heritage Tourism Engineering at Penang: Complete the Puzzle of 'The Pearl of Orient'”. Systems Engineering Procedia, 1, 358-364.

Villarreal, R. y A. van der Horst (2008). "Estrategia de competitividad turística de la República Dominicana”. ARA: Revista de Investigación en Turismo, 1 (1), 15-28.

Zhang, H. y S. Lai Lei (2012). "A Structural Model of Residents' Intention to Participate in Ecotourism: The Case of a Wetland Community". Tourism Management, 33 (4), 916-925.

Zhou, Y. et al. (2013). "Balancing the Benefits of Ecotourism and Development: The Effects of Visitor Trail-Use on Mammals in a Protected Area in Rapidly Developing China”. Biological Conservation, 165, 18-24. 